\title{
Maximum-Largest Weighted delay First Algorithm for Heterogeneous Traffic in 4G Networks
}

\author{
Elsheikh Mohamed Ahmed Esheikh, Mohamed Hadi Habaebi, Huda Adibah Mohd Ramli, \\ Mohammed H. A. Arafa, Kazi Istiaque Ahmed \\ Department of Electrical and Computer Engineering, Kulliyyah of Engineering, International Islamic University \\ Malaysia, Malaysia
}

\begin{tabular}{l} 
Article Info \\
\hline Article history: \\
Received Apr 16, 2018 \\
Revised May 2, 2018 \\
Accepted May 24, 2018 \\
\hline
\end{tabular}

Keyword:

LTEA

MAC protocol

M-LWDF

Multimedia

Scheduling

\begin{abstract}
Real time applications with strict QoS like delay sensitive applications require an advanced technology to adopt them. This is where Long Term Evolution-Advanced (LTE-A) fulfills this requirement. With this ever evolving technology the need for improvements is required. Packet scheduling is one of the important key features of LTE-A, where it dictates user selection and transmission of those user's packets based on the priority of the users to reach the receiver correctly. Packet scheduling is one mean to achieve those QoS requirements that real-time applications require. Such algorithms are HARQ Aware Scheduling Algorithm (HAS), Retransmission Aware Proportional Fair Algorithm (RAPF), Chase Combining Based Max C/I Scheduling and Maximum- Largest WeightedDealy First algorithm (MLWDF). In this paper, M-LWDF is one of the best algorithms in LTE-A which was chosen for further investigated to support QoS in high mobility environment. Packet Loss Ratio (PLR), and Mean User Throughput performance measures were used to validate the performance of M-LWDF algorithm against other algorithms using similar mobile environment. Simulation results indicate the capability of M-LWDF algorithm within the threshold of the performance measures against other benchmarks where it has demonstrated more efficiency to support and improve the performance of real-time multimedia traffic.
\end{abstract}

Copyright $\odot 2018$ Institute of Advanced Engineering and Science. All rights reserved.

Corresponding Author:

Mohamed Hadi Habaebi,

Department of Electrical and Computer Engineering,

Kulliyyah of Engineering, International Islamic University Malaysia, Malaysia

Email: habaebi@iium.edu.my

\section{INTRODUCTION}

Today, the field of Wireless communications play a vital role in shaping our everyday lives. This development in the field is propelled forward maily due to transformation of the voice telephony medium into a plethora multimedia, increasing, in the process, exponentially the demand for new wireless capacity such as the Third Generation (3G). However, a constant demand for development and better performance led to creating a new Fourth Generation (4G). The more developed verison of Long Term Evolution (LTE) Long Term Evolution-Advanced (LTE-A), which is known as release 10 and higher by the Third Generation Partnership Project (3GPP), became the leading 4G technology due to some limitations in LTE such as not having a high data rate and its vulnerability to interference and scrambling in the physical layer.

LTE-A aimed to meet the demands of higher data rate (i.e. 3/1.5 Gbs for downlink and uplink respectively) within the quality of service (QoS) required by the International Telecommunication Union Radio communication (ITU-R) with more coverage as compared to LTE. This is achieved through the usage of improved packet scheduling algorithms, such as the Carrier Aggregation (CA) technique, the enhanced usage of multi-antenna techniques and support for relay nodes [1]. Enhanced NodeB (eNB) is a combination 
of NodeB and Radio Network Controller (RNC) which interconnects the User Equipment (UE) and is able to serve more than one cell at a time while home eNB serves a femtocell. Furthermore, Enhanced Packet Core (EPC) consists of serving gateway (S-GW) for routing packets between UE and Packet Data Network (PDN), whilst Mobility Management Entity (MME) copes UE access and mobility, and PDN Gateway (PDN GW) is a gateway to PDN [2], [3].

In LTE-A, Orthogonal Frequency Division Multiple Access (OFDMA) is used for downlink transmission and Single Carrier Frequency Division Multiple Access (SC-FDMA) is used for uplink transmissions. The OFDMA symbols are grouped into Resources Blocks (RB), the RBs have a total size of $180 \mathrm{kHz}$ in the frequency domain and $1 \mathrm{~ms}$ in the time domain. Each user is allocated a number of RB and each CC contains a number of RB available for usage. However, each RB can merely be assigned to one used only during each $1 \mathrm{~ms}$ Transmission Time Interval (TTI) [4]. The LTE-A uses a simplified Evolved Universal Terrestrial Radio Access Network (E-UTRAN) architecture that only consists of eNBs' which links users to the core network and do perform all Radio Resource Management (RRM) functions. Packet scheduling is one of the key RRM functions and its uses become vital as the LTE-A delivers both delay-sensitive Guaranteed Bit Rate (GBR) and loss-sensitive Non-GBR multimedia applications using packet switching technology [1].In a non-real time (NRT) service environment, channel condition is the most common scheduling criterion, but in real-time (RT) service environment, mean user throughput, and Packet Loss Ratio (PLR) are the common scheduling criterion [5][6]. If the network overloads with packets, the scheduling algorithms plan the order of the packet transmission under various QoS requirements from different users and allocates them to different resources so that it offers larger capacity and higher data [7]. The generalized model of packet scheduling is shown in figures [1-2], while Table1 is a comparison between the specification of $3 \mathrm{G}$ and $4 \mathrm{G}$ is shown below.

Table 1. Comparison between $3 \mathrm{G}$ versus $4 \mathrm{G}[8]$

\begin{tabular}{lll}
\hline \multicolumn{1}{c}{ Specification } & \multicolumn{1}{c}{$3 \mathrm{G}$} \\
\hline Frequency Band & $1.8-2.5 \mathrm{GHz}$ & $2 \mathrm{G}$ \\
Bandwidth & $5-20 \mathrm{MHz}$ & $5-20 \mathrm{MHz}$ \\
$\begin{array}{l}\text { Data rate } \\
\text { Peak Upload Rate }\end{array}$ & Up to 2Mbps & $20 \mathrm{Mbps}$ or more \\
Peak Download Rate & $50 \mathrm{Mbit} / \mathrm{s}$ & $50 \mathrm{Mbit} / \mathrm{s}$ \\
Data Throughput & $100 \mathrm{Mbit} / \mathrm{s}$ & $1 \mathrm{Gbit} / \mathrm{s}$ \\
& Up to 3.1 Mbps & 3 to $5 \mathrm{Mbps}$ but potential estimated at a range of 10 \\
Network Architecture: & Wide Area Cell Based & to 300 Mbps \\
Switching & Circuit/Packet & Integration of wireless LAN and Wide area \\
Forward error correction (FEC) & 3G uses Turbo codes for error & $\begin{array}{l}\text { Packet } \\
\text { Concatenated codes are used for error }\end{array}$ \\
Switching Technique & correction & correctionsin4G. \\
Access & packet switching /circuit switch & packet switching, message switching \\
Services and Applications & Wideband CDMA & Multi-carrier - CDMA or OFDM(TDMA) \\
\hline
\end{tabular}

The process of scheduling a downlink LTE system is as follows: it is at $1 \mathrm{~ms}$ interval (as known as Transmit Time Interval, TTI) which consists of 2 time slots, or resource-block-pair basis (RB, one sub frame of $0.5 \mathrm{~ms}$ over $180 \mathrm{kHz}$ ). This specific TTI a user is assigned two consecutive RBs [7]. Nevertheless, once these Signal to Interference plus Noise Ratio (SINR) values in each RB are determined, it will be sent to the serving eNodeB in each TTI [9]. These SINR values that are received by each user will be used by the serving eNodeB and they will be present in each RB. This helps find out the modulation and coding scheme (MCS) that is appropriate for downlink packet transmission. After which the data rate comes into play as it is the number of bits that a user can support in two consecutive RBs in a TTI. The user's priority in channeldependent scheduling is also found here and is helped determined by the effective SINR value [10]. The rate also determines the number of bits that a user can have in two consecutive RBs in a TTI.

Moreover, a buffer is assigned to each user and any packet arriving into the buffer will be consequently time-stamped and queued to be transmitted on a First-in-First-out (FIFO) basis. This is all done at eNodeB. Furthermore, the time difference between the present time and the arrival time of a packet is computed in the queue for each packet at the eNodeB buffer. This is known as the Head of Line (HOL) packet delay. A user is separated into two groups: real-time (RT) or non-real-time (NRT) services because different delay deadlines are assigned to packets of different services. There are cases when the packet is disregarded, such as if the delay is more than the delay deadline. Each OFDM symbol is transmitted on a specific radio resource element transmits OFDM symbol of a bandwidth of $15 \mathrm{KHz}$ which lasts for 0.07 $(0.5 / 7) \mathrm{ms}$. The Multi-Input Multi-Output (MIMO) basically uses multiple antennas at transmitter (network) and receiver (terminal) side which allows simultaneous transmission of multiple data streams over one radio 
link. Either spatial multiplexing or transmit diversity MIMO scheme is selected, depending on which one is more suitable in the channel conditions present and [12].

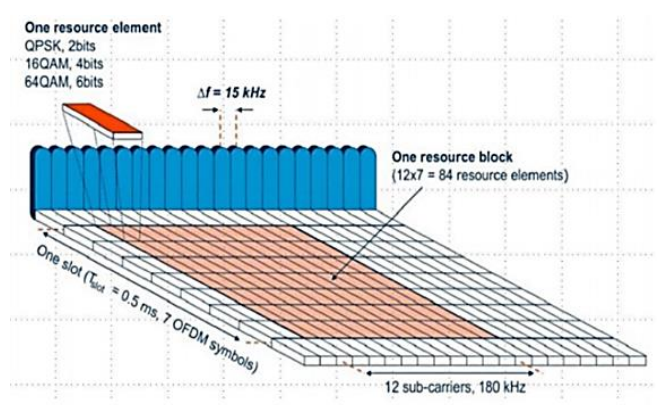

Figure 1. Radio RB component

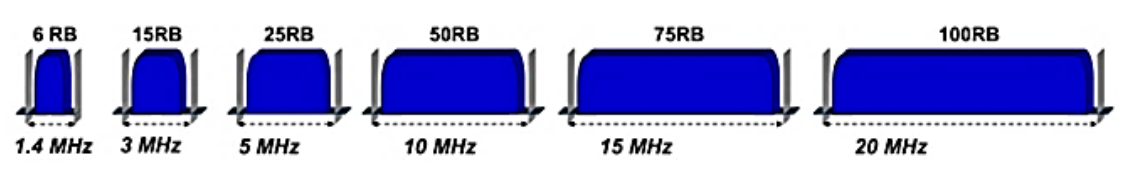

Figure 2. The LTE scalable bandwidths

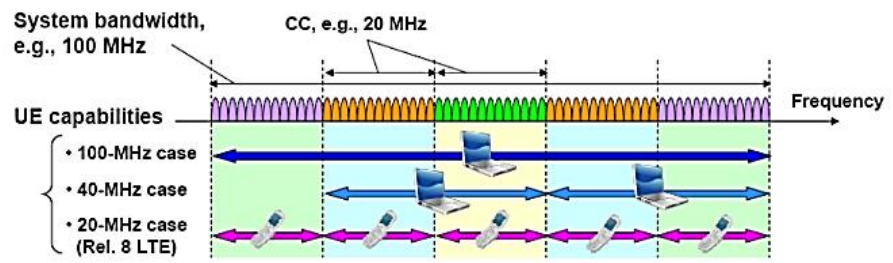

Figure 3. Wider bandwidth

A radio RB is the base unit for all the LTE radio resource activities and functionalities (see figure 1) and is made of 12 sub-carriers and 7 time-slots or $180 \mathrm{KHz}$ x $0.5 \mathrm{~ms}$ which is a combination of 84 adjacent resource elements and the possibility of forming a system bandwidth of variable size, from $1.4 \mathrm{MHz}$ with 6 RBs to $20 \mathrm{MHz}$ with $100 \mathrm{RBs}$, which is possible from the basic radio resource unit as in Figure 2. This is named a scalable bandwidth (unlike Universal Mobile Telecommunication System (UMTS)/High Speed Packet Access (HSPA), which has a fixed $5 \mathrm{MHz}$ bandwidth). Based on the size of the bits per symbol: 2 bits, 4 bits and 6 bits per symbol. LTE will use three modulation schemes: QPSK (4QAM), 16QAM, and 64QAM. Additionally, there will be link adaptation where a mobile station or eNodeB will choose the selection of modulation and channel coding schemes (MCS) to match the channel conditions; if the channel quality is good, it will use a good quality MCS to transmit at the highest data rate [11]. CA is a model where CCs are adjacent and symmetric to each other, a CC being each carrier. This is shown in Figure 3. In the case where the CCs are non-continuous and asymmetric, it is a Spectrum Aggregation. The maximum number of $\mathrm{CC}$ is 5 and an upside is that network providers can use all the available spectrums that they were assigned from the government regulator of LTE-A [13]. This is due to the spectrum flexibility.

LTE-A is backward compatible with LTE and also shares its features too, however, it has some technical improvements over LTE. According to [13], CA is the clearest way to use in order to speed up the peak data rate to meet the requirements of IMT-Advanced and an upside is that network providers can use all the available spectrums that they were assigned from the government regulator for LTE-A. LTE-A will apply MU-MIMO techniques are applied by LTE-A which is an improvement from the current SU-MIMO. All this greatly improves the peak spectrum efficiency, system data rate, capacity, as well as cell-edge user [14] and for the multi-point transmissions features in LTE-A with a single transmitter with antennas which are 
geographically separated, this will boost LTE-A as the cell-edge user is increased throughput as well as, the coverage is also increased for the deployment flexibility [15].

A HAS algorithm is used in GBR services for downlink LTE system, as it reduces the number of lost packets and keeps a low queuing delay while still holding a maximum effective user throughput [16]. Packets which require retransmission that wait in the buffer long and exceed the buffer delay threshold will be discarded, thus HAS algorithm gives highest priority for users with packets that require retransmission that has been in the buffer the longest which means those users are prioritized over other user with packets that require retransmission.

RAPF is an algorithm whose purpose is to meet the fairness standards and it uses the following equation to choose the highest priority users with new packets or retransmission:

$$
u_{i}(t)=\frac{r_{i}(t)}{R_{i}(t)}
$$

For a good mobile channel condition, the value of $r_{i}(t)$ (instantaneous data rate for user $i$ at scheduling interval $t$ ) will be high so it will result in high priority value. Vice versa, a bad mobile channel condition will result into a low value of $r_{i}(t)$ thus low priority value, which means that there will be small chance of transmitting, but $R_{i}(t)$ (average throughput of user $i$ at scheduling interval $t$ ) will be low. This leads to a high priority value. In other words, RAPF algorithm gives users good chance to transmit even though the mobile channel conditions are bad [9].

\section{MODEFIED MAXIMUM-LARGEST WEIGHTED DELAY FIRST ALGORITHM}

\subsection{Maximum-Largest Weighted Delay First Algorithm (M-LWDF)}

M-LWDF algorithm is proposed to support RT applications, it was developed for a single carrier mobile cellular system that transmits packets to the user using only one CC and across the whole bandwidth of CC. M-LWDF algorithm is suitable for satisfying the QoS stringent requirements of GBR applications that donot consider packet delay [5]. In each TTI the scheduler schedules a user that has the highest priority according to equation 2 to receive its packet in each TTI. The scheduling criteria metric $u_{i}(t)$ is defined as follows:

$$
\begin{aligned}
& u_{i}(t)=\alpha_{i} * W_{i}(t) *\left(\frac{r_{i}(t)}{R_{i}(t)}\right) \\
& \alpha_{i}=-\frac{\left(\log \delta_{i}\right)}{T_{i}} \\
& R_{i}(t)=\left(1-\frac{1}{t_{c}}\right) * R_{i}(t-1)+I_{i}(t) * \frac{1}{t_{c}} * r_{i}(t)
\end{aligned}
$$

where $u_{i}(t)$ is the priority of user $i$ at scheduling interval $t, W_{i}(t)$ is the delay of HOL packet of user $i$ at scheduling interval $t, \alpha_{i}$ is the QoS requirement of user $i, r_{i}(t)$ is the instantaneous data rate of user $i$ at scheduling interval $t, R_{i}(t)$ is the average throughput of user $i$ at scheduling interval $t, \delta_{i}$ is the application dependent Packet Loss Ratio (PLR) threshold of user $i, T_{i}$ is the application dependent buffer delay threshold of user $i, t_{c}$ is a time constant and $I_{i}(t)$ is the indicator function of the event that indicates packets of user $i$ are selected for transmission at the scheduling interval $t$ [5].

The HOL packet of a user is considered to be the packet that has stayed the longest time in the base station's buffer and the buffer delay threshold is the maximum allowable waiting period of a packet at the buffer in the base station. M-LWDF obtains a very good performance for throughput and fairness in conjunction with a relatively low PLR, because it jointly considers HOL packet delay along with PF properties. The simulation in [17] has verified that M-LWDF algorithm is efficient for usage in downlink in LTE-A for maximizing the system capacity while providing satisfactory QoS of GBR services.

\subsection{Proposed Modified M-LWDF Algorithms}

Maximum- Largest Weighted Delay First algorithm (M-LWDF) is one of the best packet scheduling algorithms for supporting GBR applications, hence, it is being considered for this research. M-LWDF algorithm was developed for mobile cellular systems that preform packet scheduling in a single CC and allocate all the available bandwidth to a user with highest priority [5]. In each time interval, the user priority is calculated according to the following formula: 


$$
u_{i}(t)=\alpha_{i} * W_{i}(t) *\left(\frac{r_{i}(t)}{R_{i}(t)}\right)
$$

Where $u_{i}(t)$ is the priority of user $i$ at scheduling interval $t, W_{i}(t)$ is the delay of HOL packet of user $i$ at scheduling interval $t, \alpha_{i}$ is the QoS requirement of user $i, R_{i}(t)$ is the average throughput of user $i$ at scheduling interval $t$ and $r_{i}(t)$ is the instantaneous data rate of user $i$ at scheduling interval $t$ [5].

Due to the characteristic of the mobile cellular systems, some packets that are transmitted to users may be received in error. As such, these packets need to be retransmitted. This research considers the situation in which the LTE-A system consists of users with new packets and packets that require retransmission.

There is more than one CCs considered in this research and packet scheduling is performed in time and frequency domain. The M-LWDF algorithm is modified in this research into three different algorithms named PSA1, PSA2 and PSA3. Each algorithm is described next. PSA1 prioritize users with retransmission of TBs over users with new transmission, PSA2 prioritize users with new packets of TBs over users with packets that require retransmission while PSA3 gives equal opportunity to all users.

In each Transmit Time Interval (TTI) and on each CC, PSA1 schedule the retransmission users first then if any resources left it will be directed to the new users. Among all the retransmission users in a cell, it selects the first retransmission users for retransmitting TB that maximize $\mu_{i, j}(t)$ in the following equation

$$
\mu_{i, j}(t)=\alpha_{i} * W_{i}(t) * \frac{r_{i, j, k}(t)}{\sum_{j=1}^{j=C C_{\max } R_{i, j}(t)}}
$$

Where $\mu_{i, j}(t)$ is the priority of user $i$ on $\mathrm{CC} j$ at scheduling interval $t, \alpha_{i}$ is the QoS requirement of user $i, W_{i}(t)$ is the delay of Head-of-Line (HOL) packet of user $i$ at $t, r_{i, j, k}(t)$ is the data rate of user $i$ on CC $j$ on $\mathrm{RB} k$ at $t, R_{i, j}(t)$ is the average throughput of user $i$ on $\mathrm{CC} j$ at $t, \mathrm{CC}_{\max }$ is the maximum number of CC available.

This equation shows that PSA1 selects retransmission user based on the data rate of each user on each CC. Then it will schedule the user on each RB and this allows multi-user diversity to be exploited for retransmission. The remaining RBs will be assigned to new users. After scheduling retransmission user, PSA1 schedule users with retransmission packets which has the highest priority based on the previous equation. It can be observed that the algorithm determines priority on each user on each RB and it aggregates the average throughput across all $\mathrm{CC}$ for each user.

PSA2 schedule the new users first based on the following equation. New user which has the highest priority will be schedule first. However, PSA2 allocates all of the available RBs to the selected user. PSA2 does not take data rate of user on each RB into consideration when determining the priority of the new user, it take the average data rate. The following equation shows PSA2 using average data rate of all RB in determining the user priority instead of the data rate in each RB. This algorithm also aggregates the average throughput across all CCs for each user. After completing scheduling the new users then it schedules the retransmission users.

$$
\mu_{i, j}(t)=\alpha_{i} * W_{i}(t) * \frac{a v g-r_{i, j}(t)}{\sum_{j=1}^{j=C C_{\max } R_{i, j}(t)}}
$$

Where $\mu_{i, j}(t)$ is the priority of user $i$ on $\mathrm{CC} j$ at scheduling interval $t, \alpha_{i}$ is the QoS requirement of user $i, W_{i}(t)$ is the delay of Head-of-Line (HOL) packet of user $i$ at $t, a v g-r_{i, j}(t)$ is the average data rate on user $i$ on $\mathrm{CC} j$ at $t, R_{i, j}(t)$ is the average throughput of user $i$ on $\mathrm{CC} j$ at $t, \mathrm{CC}_{\max }$ is the maximum number of CC available.

PSA3 proposed algorithm is similar to PSA2 as this algorithm selects user with highest priority calculated by the following equations. The difference is that PSA3 selects new packet and packets that need transmission/retransmission by applying two different equations based on the user packets type, or in simple terms the algorithm gives equal opportunity to all users. Users with the highest priority will be selected for transmission of TB. After selecting the user, PSA3 will determine the type of packets, if its retransmission packets then then required RB will be assigned randomly then the packets will be retransmitted, on the other hand if the user selected has new packets then the RB which has the best channel quality will be assigned to this packet then transmitted to the user. 


$$
\mu_{i, j}(t)=\left\{\begin{array}{c}
\alpha_{i} * W_{i}(t) * \frac{a v g-r_{i, j}(t)}{\sum_{j=1}^{j=C C_{\max } R_{i, j}(t)}} i \in \text { new user } \\
\alpha_{i} * W_{i}(t)_{\text {retrans }} * \frac{\operatorname{avg}-r_{i, j}(t)}{\sum_{j=1}^{j=C C_{\max } R_{i, j}(t)}} i \in \text { retransmission user }
\end{array}\right.
$$

Where $\mu_{i, j}(t)$ is the priority of user $i$ on $\mathrm{CC} j$ at scheduling interval $t, \alpha_{i}$ is the QoS requirement of user $i, W_{i}(t)$ is the delay of Head-of-Line (HOL) packet of user $i$ at $t, W_{i}(t)_{\text {retrans }}$ is the delay of Head-ofLine (HOL) retransmission packet of user $i$ at $t, R_{i, j}(t)$ is the average throughput of user $i$ on $\mathrm{CC} j$ at $t$, avg $-r_{i, j}(t)$ is the average data rate on user $i$ on $\mathrm{CC} j$ at $t$ and $\mathrm{CC}_{\max }$ is the maximum number of $\mathrm{CC}$ available [5].

A series of $\mathrm{C}++$ programing code is developed for computer simulation to evaluate the performance of the proposed packet scheduling algorithm PSA3and another two benchmarks PSA1 and PSA2 for validation purposes for a delay sensitive GBR applications in the downlink of LTE-A. These simulations are done within the same parameters and conditions as presented by [5] done on this subject to validate the results obtained. The parameters used on this algorithm are described as follows. All GBR users are assumed to run video streaming application with average data rates of $256 \mathrm{kbps}$. The evaluation is done within a pico cell of $500 \mathrm{~m}$ radius with an eNB located at the center of the cell. The threshold for the buffer delay is set at $20 \mathrm{~ms}$ which is within the appropriate range of the $3 \mathrm{GPP}$ recommendation [18]. The PLR threshold of $10^{-3}$ is set so the QoS requirements of the GBR application is satisfied. All of this parameters are summarized in Table 2.

Table 2. System Parameters [5], [18]

\begin{tabular}{lc}
\hline \multicolumn{1}{c}{ Parameters } & Values \\
\hline Bandwidth & $3 \mathrm{MHz}$ \\
Cell radius & $500 \mathrm{~m}$ \\
Number of RB & $15 \mathrm{RBs}$ \\
Channel quality & Error-free \\
Carrier frequency & $2 \mathrm{Ghz}$ \\
Buffer delay & $20 \mathrm{~ms}$ \\
Maximum No of users & 60 \\
Maximum No of retransmission & 3 \\
Users speed & $30 \mathrm{Km} / \mathrm{h}$ \\
\hline
\end{tabular}

Packet Loss Ratio (PLR) is defined in this paper as the ratio of the total number of dropped packets to the total number of all packets received at the serving eNodeB buffer. It is used to evaluate the QoS offered by the system at the network layer. PLR value that is acceptable varies depending on the system it's being used on, our system requires a PLR below $10^{-3}$. PLR is an important performance measure for real-time and non-real-time application due to the fact that it would standardize a value that all algorithms or codes should meet for the system to run with the maximum acceptable loss of data that it won't affect the system throughput [5] and can be mathematically represented as:

$$
P L R=\frac{\sum_{i=1}^{N} \sum_{t=1}^{T} \operatorname{Pdiscard}_{i}(t)}{\sum_{i=1}^{N} \sum_{t=1}^{T} \operatorname{Psize}_{i}(t)}
$$

Where, $i$ the total number of the dropped packets (given in bits) of user $i$ at time $t$, Psize $_{i}(t)$ : the total number of all packets (given in bits) arriving at the eNB buffer of user $i$ at time $t, T$ is the total simulation time and $N$ is the total number of users.

Mean user throughput is defined as the amount of data (packets) moved successfully from one place to another (from sender to receiver) in a given time period and its measured in bits per second (bps). It depends on the average data rate of the system and it usually degrades with time and more users in the system. The higher the throughput the better but with more users and time it falls down. One needs to maximize the mean user throughput in order to achieve the best improvement for that specific algorithm. Given that the maximum the mean user throughput to reach would be the average data rate that was used in that specific system [17]. Then the mean user throughput can be represented as:

$$
\text { Mean user throughput, } T_{u s e r}=\frac{1}{N} \frac{1}{T} \sum_{i=1}^{N} \sum_{t=1}^{T} \operatorname{Pr} x_{i}(t)
$$


Where $\operatorname{Prx}_{i}(t)$ is the total size of correctly received packets (given in bits) of user $i$ at time $t, T$ is the total simulation time and $N$ is the total number of users.

The objective of this proposed algorithm is to maximize the performance while maintaining accebtable other performance measure. The mathematical representation for maximum $T_{\text {user }}$ subject to user $(N)$ and packets received correctly $\left(\operatorname{Prx}_{i}(t)\right)$, while $\operatorname{PLR}<=10^{-3}$ and $F>=0.5$ as:

$$
T_{u s e r \max }=\frac{1}{N} \frac{1}{T} \sum_{i=1}^{N} \sum_{t=1}^{T} \operatorname{Pr} x_{i}(t), P L R \leq 10^{-3}, F \geq 0.5
$$

where $\mu_{i, j}(t)$ : the priority of user $i$ on CC $j$ at scheduling interval $t, W_{i}(t)$ is the delay of HOL packet of user $i$ at $t, \alpha_{i}$ is the QoS requirement of user $i, W_{i}(t)_{\text {retrans }}$ is the delay of Head-of-Line(HOL) retransmission packet of user $i$ at $t, R_{i, j}(t)$ is the average throughput of user $i$ on CC $j$ at $t, \operatorname{avg}-r_{i, j}(t)$ is the average data rate on user $i$ on $\mathrm{CC} j$ at $t$ and $\mathrm{CC}_{\max }$ is the maximum number of CC available [5].

Our proposed algorithm (PSA3), selects user with highest priority calculated by the following equations. It selects new packet and packets that need transmission/retransmission by applying two different equations based on the user packets type, or in simple terms the algorithm gives equal opportunity to all users. Users with the highest priority will be selected for transmission of TB. After selecting the user, PSA3 will determine the type of packets, if its retransmission packets then then required RB will be assigned randomly then the packets will be retransmitted, on the other hand if the user selected has new packets then the RB which has the best channel quality will be assigned to this packet then transmitted to the user.

$$
\mu_{i, j}(t)=\left\{\begin{array}{c}
\alpha_{i} * W_{i}(t) * \frac{\operatorname{avg}-r_{i, j}(t)}{\sum_{j=1}^{j=C C_{\max } R_{i, j}(t)} i \in \text { new user }} \\
\alpha_{i} * W_{i}(t)_{\text {retrans }} * \frac{\operatorname{avg-r_{i,j}(t)}}{\sum_{j=1}^{j=C C_{\max } R_{i, j}(t)}} i \in \text { retransmission user }
\end{array}\right.
$$

Where, $X_{i}$ : the system throughput of user $i, N$ : the total number of users, and $T$ : the total simulation time.

\section{RESULTS AND ANALYSIS}

In this results and analysis section, we tried to evaluate the performance in terms of PLR, and Mean User Throughput for our proposed algorithm.

\subsection{PLR}

Figure 3 shows the PLR of the proposed algorithm (PSA3), it can be seen that while the system at a small user number starting for zero the PLR is almost zero then it keeps on being almost zero PLR till it reaches 20 which shows that the algorithm works without any discarded packets as long the system doesn't have more than 20 users. As the channel ecomes congested, PLR then starts increasing exponentially till it breaks the threshold $\left(10^{-3}\right)$ after 22 users, this means that the system will keep on working in ideal PLR conditions as long as the number of users are maintained below $\mathrm{n}<=22$.

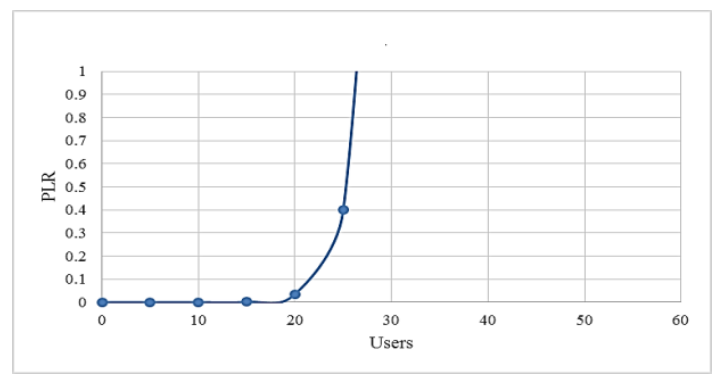

Figure 4. Packet loss ratio vs system capacity (no. of users) PSA 3

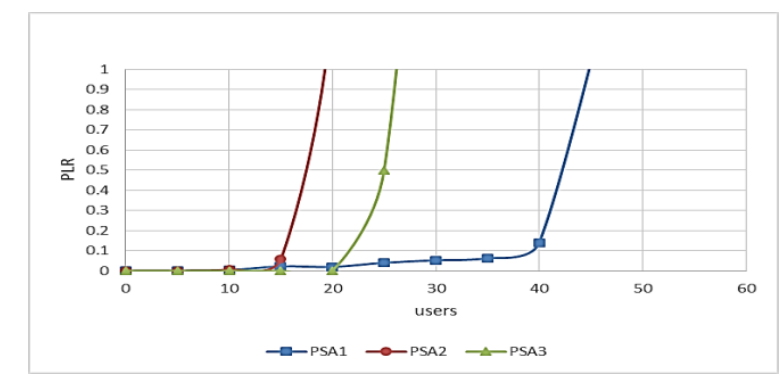

Figure 5. Packet loss ratio vs system capacity (no. of users)

Figure 4 shows PLR performance for three algorithms PSA1, PSA2, PSA3. The figure depicts that PLR degrades with increasing capacity, it can be seen that PSA2 starts with zero PLR at zero users and it 
keeps going on almost the same level till it reaches 15 users. Then it starts increasing fast and crosses the PLR threshold before it reaches the next user which makes the maximum number of users that PSA2 can work with without crossing the PLR threshold is 15 users in the cell only, while PSA1 and PSA3 still didn't cross the PLR threshold. We can see that PSA3 starts with zero PLR at zero users and it counties to be zero PLR up untill 15 users. Then it starts increasing slowly and almost not noticeable untill it reaches 20 users. Then it starts increasing exponentially to cross the threshold after 22 users which makes it the maximum system capacity for PSA3. PSA1 starts somehow similar to the other two algorithms but when it starts it stays at zero PLR till 5 users then it starts increasing slowly till it reaches around 36 users then it increases exponentially to cross the PLR threshold after 38 users which means that the maximum system capacity for PSA1 is 38 users in the cell. Table 3 compares the achieved maximum system capacity of the three benchmarked agorithms studied.

Table 3. Comparison of Achieved Maximum System Capacity

\begin{tabular}{cc}
\hline Algorithm & $\begin{array}{c}\text { Maximum system } \\
\text { capacity }\end{array}$ \\
\hline PSA1 & 38 \\
PSA2 & 15 \\
PSA3 & 22 \\
\hline
\end{tabular}

It can be observed that PSA3 has a better system capacity than PSA2 with a percentage of $46.66 \%$ while PSA1 had an improved percentage over PSA2 of $153.33 \%$. That concludes that PSA1 has the best system capacity which means better PLR performance than PSA2 and PSA3. When the number of users in a single cell is increased, there will be more packets queued in the buffer waiting for packets transmission. Due to channel impairment and insufficient of radio resource, there will be some transmission blocks (TBs) arriving at the user in error and need to be retransmitted. However, the reason why system capacity is not larger in numbers in the cell is due the fact that video streaming uses $256 \mathrm{kbps}$ which is quiet ok but doesn't allow for big amount of user packets to be transmitted. A second reason would be the high mobility of the user at $30 \mathrm{kmph}$ causes variation in CQI value. For this proposed algorithm the CQI delay was set to $20 \mathrm{~ms}$. If the user is stationary or less mobile, the value of CQI wouldn't have varied much, but due to this variation in CQI value TBs may receive the packets in error which leads to a higher value of PLR. Furthermore, a PLR threshold ratio of $10^{-3}$ is quite stringent for wireless where it is usually relaxed to double tht value (e.g., $10^{-6}$ ).

\subsection{Mean User Throughput}

Figure 5 presents the values of mean user throughput against the system capacity. Since the average data rate used in this system is $256 \mathrm{kbps}$ then the maximum mean user throughput can't be more than that as it can be observed that the highest value for mean user throughput is $256 \mathrm{kbps}$ at zero users which is the highest system capacity possible. As we observe the graph we can notice that it starts at $256 \mathrm{kbps}$ at zero users then it decreases to almost $251 \mathrm{kbps}$ and keeps a steady straight line performance of throughput as we increase the amount of users used until we reach 23 users. Then the throughput starts decreasing in a steady amount till it reaches almost $210 \mathrm{kbps}$ at 50 users.

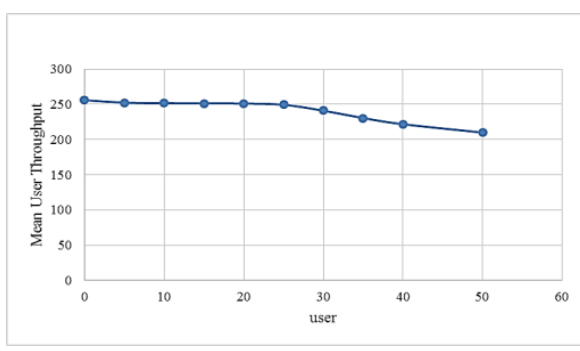

Figure 6. Mean user throughput(kbps) vs system capacity for PSA 3

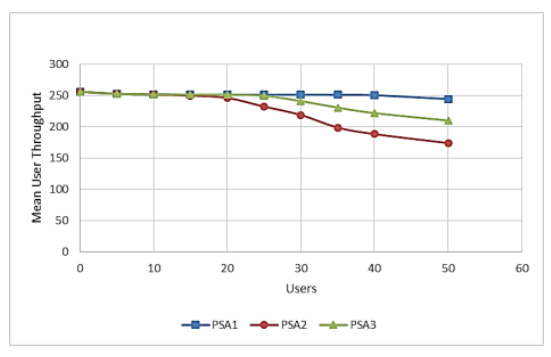

Figure 7. Mean user throughput (kbps) vs system capacity

Figure 6 shows the mean user throughput against system capacity that is obtained for PSA1 while Figure 7 benchmarks the mean user throughput of the three scheduling algorithms. As we can see all algorithms start at $256 \mathrm{kbps}$ at zero users and then decrease little bit to almost 251 and maintain it till 17 
users for PSA2 then PSA2 starts decreasing more than the other two so that makes PSA2 the algorithm with the worst throughput out of all the three as it degrades rapidly with increasing users till it reaches almost 175 kbps at 50 users.Meanwhile, PSA1 maintains approximately $251 \mathrm{kbps}$ throughput with minor decrease after 40 users to reach almost $245 \mathrm{kbps}$ at 50 users. PSA3 maintains approximately $251 \mathrm{kbps}$ throughput up till 23 users then it starts decreasing in a steady amount to reach almost $210 \mathrm{kbps}$ at 50 users. This means PSA1 is the best performer in terms of mean user throughput.

However, PSA2 shows the least throughput values out of all which is expected since it focuses on users with new packets and leaves the other users to wait either for their turn if it came up or to be discarded for vaulting the buffer delay threshold. Second comes PSA3 which not shocking giving that the PLR and Mean User Through values come second to PSA1, but still it's much more fair than PSA2. Now PSA1 comes on top because it has the best PLR and Mean User Throughput values throughout the whole system run. Regardless of this ranking all of three algorithms do not cross the threshold which means they are fair to their users. One can conclude that this algorithm works and meets the two performance measures. In terms of PLR the system gives perfect output that meets the PLR requirements which is not cross the $10^{-3}$ threshold as long as $\mathrm{n}<=22$ users. When it comes to mean user throughput it gives almost perfect throughput up till 25 users. However, even then the decrease after that is not big in value and as observed from the graphs this algorithm is fair to all users. Referring to the obtained results it can be mentioned that PSA3 has much better improvement over its successor PSA2 $46 \%$ in PLR, and 12\% in Mean User Throughput. While PSA3 still relatively great packet scheduling algorithm, it couldn't beat PSA1 in terms of performance measures. PSA2 performed the worst because it prioritizes new users and it allocated all available RBs to the scheduled user leaving the rest to wait in the buffer, thus great PLR value. Then comes PSA1 with it's great PLR, and Mean User Throughput performance that is because it schedules each user on its RB, hrnce, leading to multi-user diversity. Table 3 compares the achievable PLR for our PSAs to recent work in [19]. It shows that PSA1 achieves much better performance in terms of PLR for the same type of traffic while the sudden exponential increase sue to 10-3 stringent threshold is evident.

Table 3: PLR (\%) Comprison between PSAs and Recent Works

\begin{tabular}{ccccc}
\hline No. Users & PSA1 & PSA2 & PSA3 & {$[19]$} \\
\hline 10 & 1 & 1 & 0 & 0.3 \\
20 & 0.01 & 100 & 0 & 0.4 \\
30 & 0.05 & - & 100 & 0.5 \\
40 & 0.15 & - & - & 0.55 \\
50 & 100 & - & - & -
\end{tabular}

\section{CONCLUSION}

This research aims to find a packet scheduling algorithm over M-LWDF to improve the real time multimedia performance in LTE-A. Three different PSAs were proposed based on MLWDF modifications and their performance was benchmarked and copared. PSA1 prioritized users with packets that require retransmission while scheduling each user on each RB. PSA2 prioritized new users as it allocated all available RBs to the user with the highest priority based on its equation and PSA3 gives equal opportunity to all users whether it's a new user or user that requires retransmission and it allocates all RBs to the scheduled user. It can be concluded that the proposed PSA3 algorithm is a good algorithm given its PLR, and Mean User Throughput values in comparison to other two PSAs. It does satisfy the QoS requirements for real time multimedia applications, which makes a great real-time multimedia performance.Nevertheless, all packet scheduling algorithms need to compete with the ever evolving requirements to suit the futuristic technologies.

\section{REFERENCES}

[1] A. Ghosh, R. Ratasuk, B. Mondal, N. Mangalvedhe, and T. Thomas, "LTE-advanced: Next-generation wireless broadband technology," IEEE Wirel. Commun., vol. 17, no. 3, pp. 10-22, 2010.

[2] G. Yuan, X. Zhang, W. Wang, and Y. Yang, "Carrier aggregation for LTE-advanced mobile communication systems," IEEE Commun. Mag., vol. 48, no. 2, pp. 88-93, 2010.

[3] M. Al-Shibly, Mohammed Abduljawad and Habaebi, Mohamed Hadi (2012) Carrier aggregation in long term evolution advanced. In: 2012 IEEE Control and System Graduate Research Colloquium (ICSGRC 2012), 16-17 July 2012, Shah Alam, Selangor.

[4] H. A. M. Ramli, R. Basukala, K. Sandrasegaran, and R. Patachaianand, "Performance of well known packet scheduling algorithms in the downlink 3GPP LTE system," Proc. - MICC 20092009 IEEE 9th Malaysia Int. Conf. Commun. with a Spec. Work. Digit. TV Contents, no. December, pp. 815-820, 2009. 
[5] H. A. M. Ramli, "Performance of maximum-largest weighted delay first algorithm in long term evolution-advanced with carrier aggregation," IEEE Wirel. Commun. Netw. Conf. WCNC, vol. 2, pp. 1415-1420, 2014.

[6] Habaebi, Mohamed Hadi and Chebil, Jalel and Al-Sakkaf, A.G. and Dahawi, T. H. (2013) Comparison between scheduling techniques in long term evolution. International Islamic University Malaysia Engineering Journal, 14 (1). pp. 66-75. ISSN 1511-788X

[6] T.-Y. Tsai, Y. Chung, and Z. Tsai, "Introduction to Packet Scheduling Algorithms for Communication Networks," Commun. Netw., no. September, pp. 264-287, 2010.

[8] E. Dahlman, S. Parkvall, J. Sköld, and P. Beming, 3 G Evolution: HSPA and LTE for Mobile Broadband. Academic Press, Inc., 2011.

[9] L. X. Lin, Y. A. Liu, F. Liu, G. Xie, K. M. Liu, and X. Y. Ge, "Resource scheduling in downlink LTE-advanced system with carrier aggregation," J. China Univ. Posts Telecommun., vol. 19, no. 1, p. 44-49,123, 2012.

[10] J. S. and P. B. Erik Dahlman, Stefan Parkvall, "3G Evolution: HSPA and LTE for Mobile Broadband," Elsevier, no. 1, pp. 1-5, 2014.

[11] Rayal Frank, "An overview of the LTE physical layer. EE Times," EETimes. [Online]. Available: https://www.eetimes.com/document.asp?doc_id=1278096. [Accessed: 14-Apr-2018].

[12] C. S. Park, Y. P. E. Wang, G. Jöngren, and D. Hammarwall, "Evolution of uplink MIMO for LTE-advanced," IEEE Commun. Mag., vol. 49, no. 2, pp. 112-121, 2011.

[13] H. Holma and A. Toskala, LTE for UMTS: Evolution to LTE-Advanced, Second Edi. John Wiley and Sons, Ltd., 2011.

[14] M. Morant, A. Macho, and R. Llorente, "Optical Fronthaul of LTE-Advanced MIMO by Spatial Multiplexing in Multicore Fiber," Opt. Fiber Commun. Conf. 2015, vol. OSA Techni, 2015.

[15] B. Clerckx, A. Lozano, S. Sesia, C. van Rensburg, and C. Papadias, "3GPP LTE and LTE-Advanced," EURASIP J. Wirel. Commun. Netw., vol. 2009, no. 1, p. 472124, 2009.

[16] H. A. M. Ramli, K. Sandrasegaran, R. Basukala, and T. S. Afrin, "HARQ aware scheduling algorithm for the downlink LTE system," 2011 4th Int. Conf. Model. Simul. Appl. Optim. ICMSAO 2011, pp. 2-5, 2011.

[17] H. A. M. Ramli, K. Sandrasegaran, A. F. Ismail, S. A. Latif, and F. N. M. Isa, "A simulation tool for downlink long term evolution-advanced," Res. J. Appl. Sci. Eng. Technol., vol. 8, no. 19, pp. 2032-2041, 2014.

[18] H. A. M. Ramli, F. N. M. Isa, A. F. Ismail, M. K. Hasan, and W. Hashim, "Impact of outdated CQI report on adapted well-known packet scheduling algorithm when streaming video," Int. Conf. Sp. Sci. Commun. Iconsp., vol. 2015-Septe, pp. 383-388, 2015.

[19] S. Fouziya Sulthana and R. Nakkeeran, "Study of Downlink Scheduling Algorithms in LTE Networks," Journal of Networks, vol. 9, No. 12, 2014, pp. 3381-3391, 2014. 